\title{
One, two, or three stars? An investigation of an unusual eclipsing binary candidate undergoing dramatic period changes ${ }^{\star}$
}

\author{
M. E. Lohr ${ }^{1}$, A. J. Norton ${ }^{1}$, U. C. Kolb ${ }^{1}$, and D. R. S. Boyd ${ }^{2}$ \\ ${ }^{1}$ Department of Physical Sciences, The Open University, Walton Hall, Milton Keynes MK7 6AA, UK \\ e-mail: Marcus.Lohr@open.ac.uk \\ 2 The British Astronomical Association, Burlington House, Piccadilly, London W1J 0DU, UK
}

Received 26 July 2013 / Accepted 29 August 2013

\section{ABSTRACT}

\begin{abstract}
We report our investigation of 1SWASP J234401.81-212229.1, a variable with a 18461.6 s period. After identification in a 2011 search of the SuperWASP archive for main-sequence eclipsing binary candidates near the distribution's short-period limit of $\sim 0.20 \mathrm{~d}$, it was measured to be undergoing rapid period decrease in our earlier work, though later observations supported a cyclic variation in period length. Spectroscopic data obtained in 2012 with the Southern African Large Telescope did not, however, support the interpretation of the object as a normal eclipsing binary. Here, we consider three possible explanations consistent with the data: a single-star oblique rotator model in which variability results from stable cool spots on opposite magnetic poles; a two-star model in which the secondary is a brown dwarf; and a three-star model involving a low-mass eclipsing binary in a hierarchical triple system. We conclude that the latter is the most likely model.
\end{abstract}

Key words. stars: individual: 1SWASP J234401.81-212229.1 - stars: variables: general - binaries: close - binaries: eclipsing

\section{Introduction}

The object 1SWASP J234401.81-212229.1 (J2344) was identified as a candidate W UMa-type (contact) eclipsing binary in Norton et al. (2011), primarily on the basis of light curve shape. Using observations from the SuperWASP archive (Pollacco et al. 2006), a best period of $0.21367 \mathrm{~d}$ was found ${ }^{1}$, giving it immediate interest as being very close to the observed lower limit in the period distribution of main sequence binaries of $\sim 0.2 \mathrm{~d}$ (Rucinski 1992). Lohr et al. (2012) then found evidence of substantial period changes in J2344, which suggested rapid period decrease on the basis of the first four years of SuperWASP data, implying a stellar merger within 40000 years at most. However in Lohr et al. (2013a), which found the object's period as $18461.639 \pm 0.0005 \mathrm{~s}(0.21367638 \mathrm{~d})$, more recently-available SuperWASP observations supported a subsequent increase in period: J2344 currently appears to be undergoing dramatic and approximately sinusoidal variations in period length.

Two other objects from the Lohr et al. (2013a) collection of 143 SuperWASP candidate eclipsing binaries with periods below 20000 s have since been followed up spectroscopically, and in spite of relatively low signal-to-noise $(\mathrm{S} / \mathrm{N})$ spectra, were confirmed as low-mass double-lined spectroscopic and eclipsing binaries (Lohr et al. 2013b). Therefore, with high-resolution spectra, the prospects seemed good for confirming J2344 as a

\footnotetext{
* Table 2 is only available at the CDS via anonymous ftp to cdsarc.u-strasbg.fr (130.79.128.5) or via http://cdsarc.u-strasbg.fr/viz-bin/qcat?]/A+A/558/A71 1 The corresponding object ASAS J234402-2122.5 found from ASAS observations is listed in the AAVSO International Variable Star Index as a W UMa-type eclipsing binary with a period of $0.2136764 \mathrm{~d}$; in the ASAS Catalog of Variable Stars as a semi-detached eclipsing binary, period $0.213678 \mathrm{~d}$; and in the Machine-learned ASAS Classification Catalog as a $\delta$ Scuti pulsating variable with period $0.10684 \mathrm{~d}$.
}

binary system, determining its parameters and identifying the cause of its period variation. Observations were made with the Southern African Large Telescope (SALT) in mid-2012, from which we hoped to extract radial velocities (RVs) for the system components. Moreover, additional photometric observations of J2344 were made during late 2012, with a view to measuring more recent eclipse timings.

Here we report the surprising results of the follow-up observations: that $\mathrm{J} 2344$ does not appear to be a straightforward eclipsing binary system. It remains a mysterious object, though certain explanations are supported by the data while others are ruled out. We consider three possible models, and identify one as most plausible on current evidence. Whatever the true nature of this object, it is hoped that this exploration will be of value for studies of low-mass stars and variables in general.

\section{Observations}

\subsection{Photometry}

The SuperWASP archive contains 21727 photometric points for J2344, obtained between 15 May 2006 and 2 August 2011. The fluxes, approximately corresponding to the Johnson $V$ band, were corrected by Sys-Rem (Tamuz et al. 2005; Mazeh et al. 2006), and were extracted using a 3.5 pixel-radius photometric aperture (the middle of SuperWASP's three photometric apertures), corresponding to $477^{\prime \prime} 95$. Figure 1 shows the local star field, including two nearby sources evaluated for their possible contribution to the observed SuperWASP light curve. Periods and period changes were measured using a custom IDL program, described in Lohr et al. (2013a), resulting in a high-precision phase-folded light curve.

Additional photometric observations were made of J2344 and nearby sources by D. Boyd in the southern UK, for $0.5 \mathrm{~h}$ 


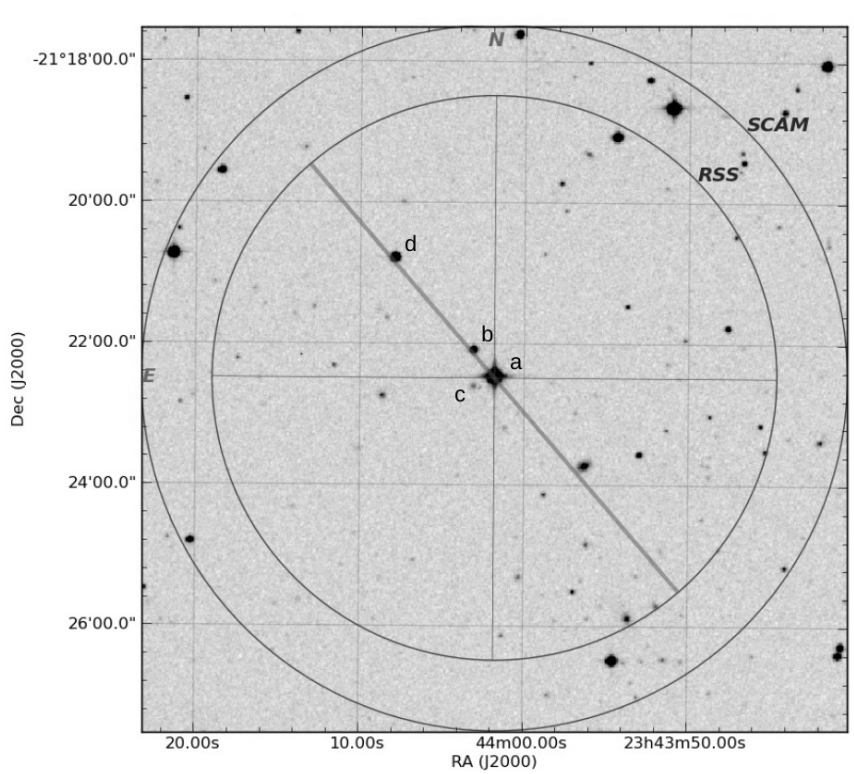

Fig. 1. Local star field of J2344 (a). The outer circle shows the field of view of the SALTICAM imager; the inner is that of the Robert Stobie Spectrograph (RSS). The diagonal line shows the angle of the spectrograph slit, chosen to include potential comparison stars b and d. Sources b and c would have fallen within the SuperWASP photometric aperture for $\mathrm{J} 2344$.

on 18 December and $1.1 \mathrm{~h}$ on 29 December 2012. A $0.35 \mathrm{~m}$ telescope with Starlight Xpress SXV-H9 CCD was used (pixel size $12.9 \mu \mathrm{m}=1$ '.'2). On 18 December, the average full width at half maximum was 3". 6 , air mass 3.4 , and exposure duration $30 \mathrm{~s}$; on 29 December the corresponding values were 5".6, 5.7 and $60 \mathrm{~s}$; both nights were affected by moonlight and low-altitude haze. One additional eclipse timing (HJD 2456291.33132) was determined, and the variability and magnitudes of several sources in the vicinity of $\mathbf{J} 2344$ were measured, using comparison stars GSC 06410-00829, GSC 06410-01027 and GSC 06410-00871 (magnitudes obtained from AAVSO APASS survey).

\subsection{Spectroscopy}

53 long-slit spectra were taken for J2344 according to an automated schedule, by duty astronomers at SALT (Buckley et al. 2006), using the PG1800 grating on the RSS (Burgh et al. 2003) on 1 June $(16 \times 60 \mathrm{~s}), 2$ July $(16 \times 60 \mathrm{~s}), 1$ August $(4 \times 60 \mathrm{~s})$ and 3 August $(16 \times 60 \mathrm{~s} ; 1 \times 12 \mathrm{~s})$. By chance there was substantial overlap in the phases covered by the second and fourth nights of observation; a total phase coverage of $\sim 22 \%$ was achieved. The slit, with a width of 0.9 , was intended to be aligned at $35.75^{\circ}$ to capture two nearby stars for potential comparison with J2344 (see Fig. 1), but this was achieved to varying extents during the second, third and fourth nights, and not at all during the first night. A wavelength range of $\sim 5800-7100 \AA$ was covered, to include the $\mathrm{NaI} \mathrm{D}$ doublet, $\mathrm{H} \alpha$ and a large number of narrow metal lines characteristic of cool stars.

Primary reduction was carried out by the SALT pipeline, using the PySALT software package ${ }^{2}$ (Crawford et al. 2010). This included fidelity checking, gain and cross-talk correction, overscan bias subtraction and amplifier mosaicking. Master bias subtraction is not suggested for SALT data; also, flatfielding, cosmic-ray rejection and fringe subtraction were not

\footnotetext{
2 http://pysalt.salt.ac.za/
}

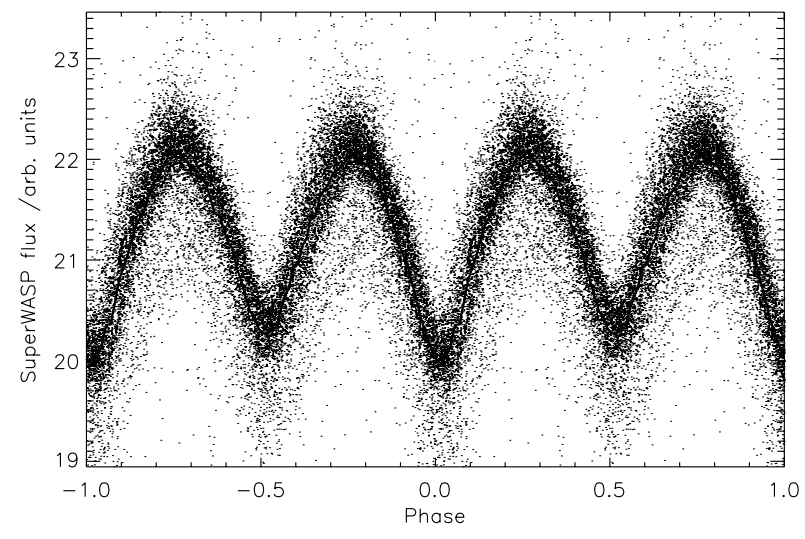

Fig. 2. SuperWASP light curve for J2344, folded at period of 18461.639 s, with binned mean curve overplotted.

implemented in the pipeline at the time, pending calibration. After initial attempts to use unflattened spectra, a master flat was constructed as a median of 10 flats supplied with the August spectra, and applied to all program images. Spectra were then optimally extracted using standard IRAF routines (which effectively cleaned out cosmic rays), and calibrated using neon arc lamp exposures. A resolution of $\sim 0.41 \AA$ per pixel was obtained.

In the apparent absence of visible line splitting or shifting in the spectra, no data-internal determination of phase was possible. Therefore phases were assigned to the spectra using a SuperWASP ephemeris in combination with D. Boyd's more recent eclipse timing. The source's spectral type was confirmed by cross-correlation using the IRAF task FXCOR, with comparison templates drawn from the Indo-US Library of Coudé Feed Stellar Spectra (Valdes et al. 2004), which uses a comparable resolution (0.44 $\AA$ ) and wavelength range (3460-9464 $\AA$ ). Crosscorrelation with a program spectrum of phase 0 was used to measure RVs, since the assumed two component spectra would be coincident during the primary eclipse.

\section{Results}

Figure 2 shows J2344's light curve, folded at its optimal period of $18461.639 \mathrm{~s}$. There is a small but consistent difference in the depths of primary and secondary minima, and continuous light variation, explaining its preliminary identification as an eclipsing binary in thermal contact. However, we may note the small amplitude of variation relative to the maximum or "outof-eclipse" flux level of $\sim 22$ units $(\sim 11.6 \mathrm{~V} \mathrm{mag})$ : only about $1 / 11$ of the light is lost during the assumed primary eclipse. This would imply a low angle of inclination of the system, a low mass ratio, and/or a third light entering the aperture.

Lohr et al. (2013a) discusses the period changes we observed in J2344, and its Figs. 5 and 6 illustrate the best linear and quadratic fits to the data. The first four years suggested a rapid period decrease (reflected in an $\mathrm{O}-\mathrm{C}$ parabola opening downwards), but the most recent year of SuperWASP data conflicted with this model $\left(\chi^{2}=17.83\right)$; assuming a half-cycle error in the primary minimum fits of the final year's data improved the quadratic fit $\left(\chi^{2}=10.86\right)$ but left Year 4 now visibly discrepant. Here, Fig. 3 gives the best sinusoidal fit to the SuperWASP data and the additional observation of a primary minimum from 2012; this provides an optimal model $\left(\chi^{2}=2.82\right)$, and suggests a meta-period for $\mathrm{J} 2344$ of $4.19 \pm 0.04$ years, with an amplitude of $631 \pm 11 \mathrm{~s}$. The observed times of minima are listed in Table 2 (only available in electronic form at the CDS). 


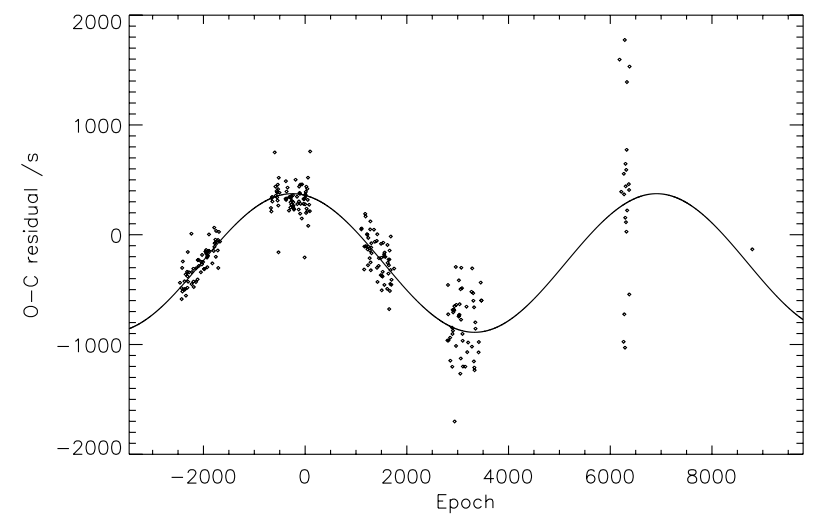

Fig. 3. O-C diagram for $\mathrm{J} 2344$, following subtraction of best linear fit. Best sinusoidal fit is overplotted (meta-period $4.19 \mathrm{y} ; \chi^{2}=2.82$ ). Uncertainties on individual points are not shown for clarity, but are typically of the order of $\pm 100 \mathrm{~s}$. The final point, around epoch 9000 , corresponds to the independent observation of D. Boyd, and has an uncertainty of $\pm 60 \mathrm{~s}$.

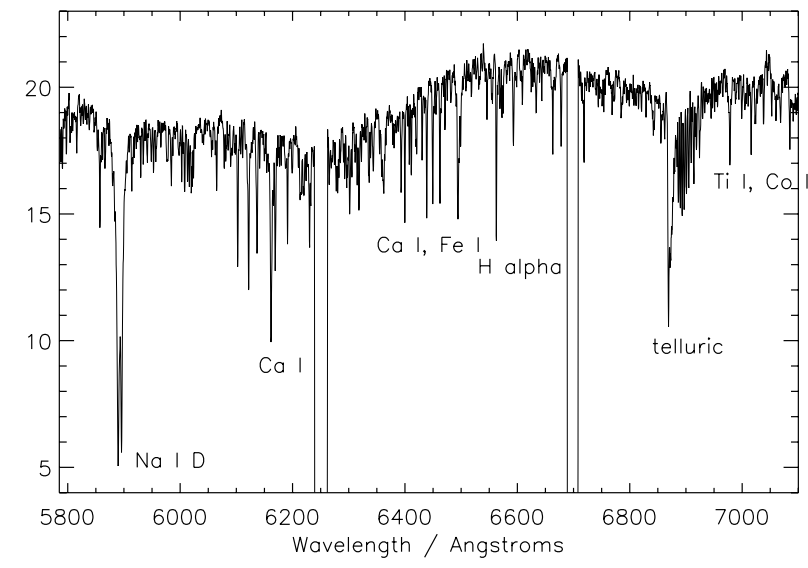

Fig. 4. SALT spectrum at phase 0.588. The gaps around 6250 and $6700 \AA$ A correspond to the boundaries of the three CCDs.

Figure 4 shows an example full extracted and calibrated spectrum for $\mathrm{J} 2344$. The best-matching comparison spectra were around K5V (temperatures between 4000 and $4500 \mathrm{~K}$ ), achieving cross-correlation peak heights in excess of 0.95 . However, to our surprise, little to no splitting or even shifting of the many well-defined absorption lines observed was apparent to the eye, as would be expected in a close, short-period eclipsing binary (Fig. 5). Only for spectra near phase 0.25 is there any suggestion of a leftward shift, and unfortunately, these spectra are by far the faintest of all four sets of observations, reducing their reliability. Moreover, an approximate light curve extracted from the spectra themselves (by fitting a spline to each continuum and evaluating it at a given wavelength) did not reflect the SuperWASP light curve at all, being apparently dominated by systematic effects.

Table 1 gives the heliocentric times, estimated phases and RVs for J2344's spectroscopic observations. Only one clear cross-correlation peak was seen for each spectrum, rather than the two that we would expect for an eclipsing binary. (Repetition of the measurements using a template $\mathrm{K} 5 \mathrm{~V}$ spectrum produced very similar results, apart from a systematic offset due to relative centre-of-mass system velocities). Figure 6 plots the resulting RV curve against phase. We may note that the amplitude of variation is very small: only $\pm 5 \mathrm{~km} \mathrm{~s}^{-1}$, where we might expect values of tens or hundreds of $\mathrm{km} \mathrm{s}^{-1}$. Also, such trends as are suggested over the orbital cycle do not seem to correspond

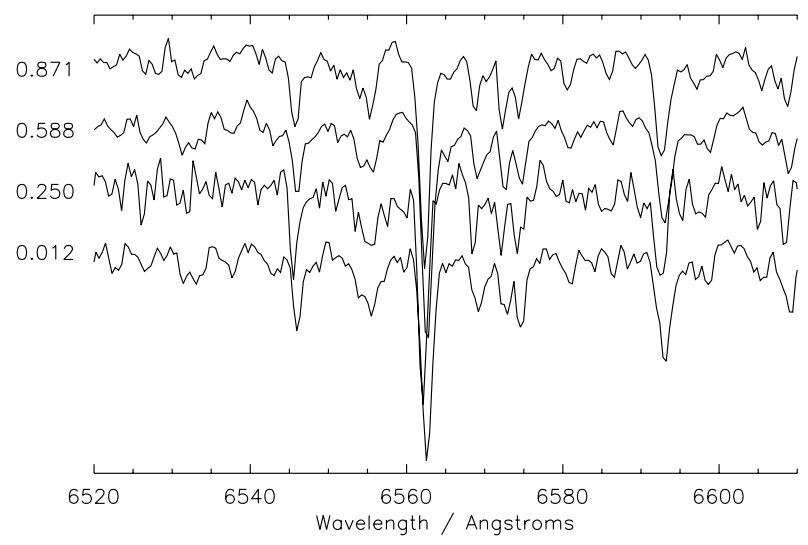

Fig. 5. Sections of SALT spectra around $\mathrm{H} \alpha$ line at $6562.8 \AA$, selected from the four nights of observation, and covering as wide a phase range as possible (phases shown on left).

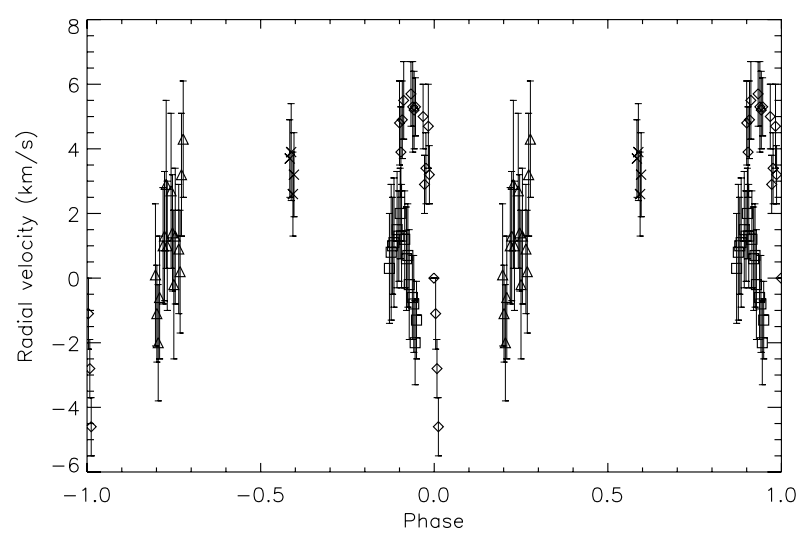

Fig. 6. RV curve for J2344. First night's observations are plotted with triangles; second night: squares; third night: crosses; fourth night: diamonds.

to expected velocity changes for either component of a spectroscopic binary: some sort of maximum appears around phase 0.9 during the region of overlapping phase coverage, but this is when we expect the primary's RV to be decreasing, and the secondary's to be increasing; phase 0.25 should correspond to the primary's minimum RV and the secondary's maximum, but actually shows a rising trend in our plot.

\section{Discussion}

Our spectroscopic results were not as expected given the photometric data for J2344. Its SuperWASP light curve (and indeed, the ASAS light curve of the corresponding source) strongly suggests a very short period eclipsing binary in contact configuration, like those of 1SWASP J150822.80-054236.9 and 1SWASP J160156.04+202821.6, which were recently confirmed as spectroscopic double-lined and eclipsing binaries, on the basis of fairly low-resolution INT spectra, and modelled as contact systems (Lohr et al. 2013b). However, the SALT spectra for J2344 showed little to no evidence for line splitting or shifting, being instead strongly consistent with a single, stable mid-K star.

To confirm our impression of the inconsistency of the photometric and spectroscopic results, modelling was carried out using the eclipsing binary modelling software PHOEBE (Prša $\&$ Zwitter 2005), built upon the code of Wilson \& Devinney (1971). Figures 7 to 10 illustrate the best light curve and RV 
Table 1. Summary of spectroscopic observations and RVs for J2344

\begin{tabular}{lccc|cccc}
\hline \hline $\begin{array}{l}\text { HJD } \\
-2450000\end{array}$ & Phase & $\begin{array}{c}R V \\
\left(\mathrm{~km} \mathrm{~s}^{-1}\right)\end{array}$ & $\begin{array}{c}\delta R V \\
\left(\mathrm{~km} \mathrm{~s}^{-1}\right)\end{array}$ & $\begin{array}{c}\text { HJD } \\
-2450000\end{array}$ & Phase & $\begin{array}{c}R V \\
\left(\mathrm{~km} \mathrm{~s}^{-1}\right)\end{array}$ & $\begin{array}{c}\delta R V \\
\left(\mathrm{~km} \mathrm{~s}^{-1}\right)\end{array}$ \\
\hline 6079.6205 & 0.197 & 0.1 & 2.2 & 6110.5461 & 0.928 & -0.2 & 1.7 \\
6079.6214 & 0.201 & -1.1 & 1.5 & 6110.5480 & 0.937 & -0.6 & 1.3 \\
6079.6222 & 0.205 & -2.0 & 1.8 & 6110.5489 & 0.941 & -0.8 & 1.5 \\
6079.6231 & 0.209 & -0.6 & 1.9 & 6110.5498 & 0.945 & -2.0 & 1.3 \\
6079.6252 & 0.219 & 1.0 & 1.8 & 6110.5506 & 0.949 & -1.3 & 1.2 \\
6079.6261 & 0.223 & 1.3 & 2.0 & & & & \\
6079.6270 & 0.228 & 2.9 & 2.6 & 6141.4557 & 0.584 & 3.7 & 1.2 \\
6079.6278 & 0.231 & 1.0 & 2.0 & 6141.4566 & 0.588 & 3.9 & 1.5 \\
6079.6300 & 0.242 & 2.7 & 2.4 & 6141.4575 & 0.593 & 2.6 & 1.3 \\
6079.6309 & 0.246 & 1.4 & 1.8 & 6141.4583 & 0.596 & 3.2 & 1.3 \\
6079.6317 & 0.250 & -0.2 & 2.3 & & & & \\
6079.6326 & 0.254 & 1.3 & 2.1 & 6143.4463 & 0.900 & 4.8 & 1.3 \\
6079.6348 & 0.264 & 0.9 & 2.0 & 6143.4471 & 0.904 & 3.9 & 1.4 \\
6079.6357 & 0.268 & 0.2 & 1.9 & 6143.4480 & 0.908 & 4.9 & 1.2 \\
6079.6366 & 0.272 & 3.2 & 1.9 & 6143.4489 & 0.912 & 5.5 & 1.2 \\
6079.6375 & 0.277 & 4.3 & 1.8 & 6143.4534 & 0.933 & 5.7 & 1.0 \\
& & & & 6143.4543 & 0.938 & 5.3 & 1.4 \\
6110.5340 & 0.871 & 0.3 & 1.7 & 6143.4552 & 0.942 & 5.2 & 1.2 \\
6110.5349 & 0.876 & 0.8 & 2.1 & 6143.4560 & 0.946 & 5.3 & 0.9 \\
6110.5358 & 0.880 & 1.0 & 1.5 & 6143.4607 & 0.968 & 5.0 & 1.0 \\
6110.5367 & 0.884 & 1.1 & 2.0 & 6143.4616 & 0.972 & 2.9 & 0.9 \\
6110.5387 & 0.893 & 1.5 & 1.8 & 6143.4625 & 0.976 & 3.4 & 1.1 \\
6110.5396 & 0.898 & 1.3 & 1.4 & 6143.4640 & 0.983 & 4.7 & 1.3 \\
6110.5405 & 0.902 & 2.0 & 1.4 & 6143.4647 & 0.986 & 3.2 & 0.9 \\
6110.5413 & 0.906 & 1.3 & 1.6 & 6143.4675 & 0.999 & 0.0 & 0.0 \\
6110.5434 & 0.915 & 1.2 & 1.5 & 6143.4684 & 0.004 & -1.1 & 1.1 \\
6110.5443 & 0.920 & 0.6 & 1.6 & 6143.4693 & 0.008 & -2.8 & 0.9 \\
6110.5452 & 0.924 & 0.7 & 1.6 & 6143.4702 & 0.012 & -4.6 & 0.9 \\
\hline & & & & & & &
\end{tabular}

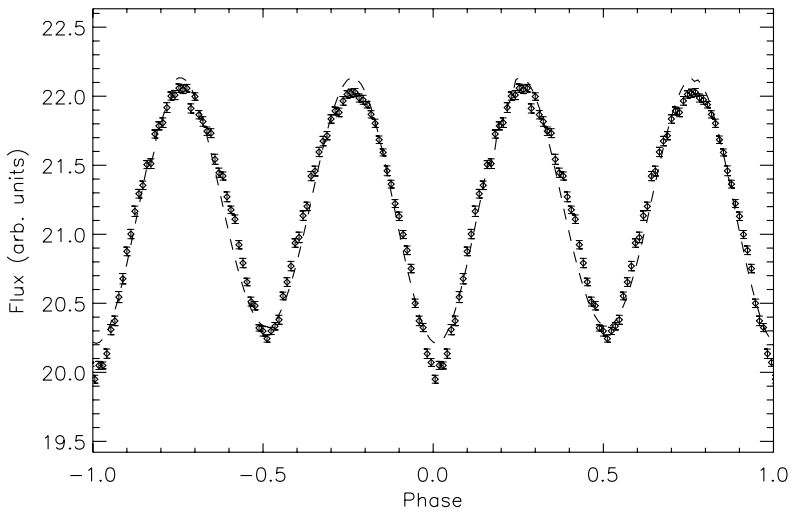

Fig. 7. Best light curve fit for PHOEBE model 1 of eclipsing binary assuming primary has mass consistent with $\mathrm{K} 5 \mathrm{~V}$ star, and secondary is massive enough to burn hydrogen $\left(i=44^{\circ}, q=0.11, M_{1}=0.73 M_{\odot}\right.$, $\left.M_{2}=0.08 M_{\odot}\right)$. Binned input SuperWASP light curve shown with diamonds and uncertainty bars; fit with dotted line.

curve fits for two models: one optimising light curve fit and the second optimising RV curve fit. Input parameters of semimajor axis and mass ratio were constrained by the requirements that the more massive star in the assumed binary be consistent with a $\mathrm{K} 5 \mathrm{~V}$ spectrum, and its companion be massive enough to burn hydrogen, so that the system contains two main sequence stars. It may be seen that, with the minimum mass ratio $q=0.11$, the observed light curve can be tolerably reproduced with a moderate angle of inclination $i=44^{\circ}$ (Fig. 7), but the corresponding primary RVs are then about three times larger than observed (Fig. 8). However, if $i$ is reduced far enough to bring the modelled RV curve into the observed range (Fig. 10),

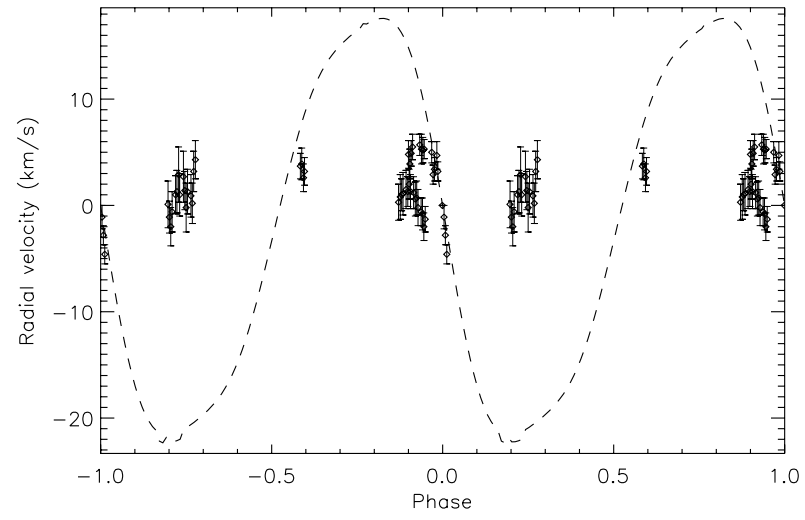

Fig. 8. Best primary RV curve fit for PHOEBE model 1 (parameters as for Fig. 7). SALT RV curve shown with diamonds and uncertainty bars; fit with dotted line.

the corresponding light curve model has far too small an amplitude (Fig. 9). Higher mass ratios fail to reproduce either light curve or RV curve, at any angle of inclination. Therefore we conclude that the observed photometry and spectroscopy, taken together, are incompatible with any low-mass eclipsing binary composed of main sequence stars.

One initial explanation considered was that J2344 was not in fact the source of the light variability observed by SuperWASP (and ASAS). Objects b and c, shown in Fig. 1, are close enough to J2344 to have fallen within the same SuperWASP photometric aperture. Could one of these be the expected eclipsing binary? Object b was captured within the SALT slit during three nights of observations, so its spectrum was also extracted and reduced. Although much fainter and noisier than 


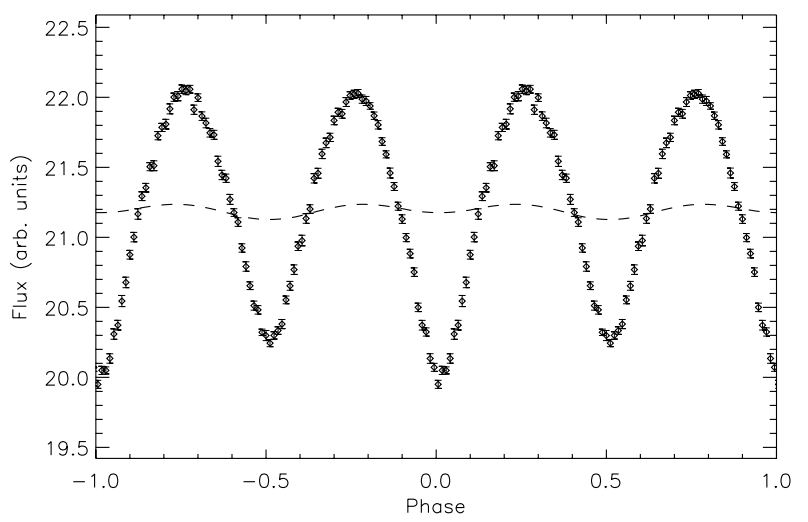

Fig. 9. Best light curve fit for PHOEBE model 2 of eclipsing binary assuming primary has mass consistent with $\mathrm{K} 5 \mathrm{~V}$ star, and secondary is massive enough to burn hydrogen $\left(i=10^{\circ}, q=0.11, M_{1}=0.73 M_{\odot}\right.$, $\left.M_{2}=0.08 M_{\odot}\right)$.

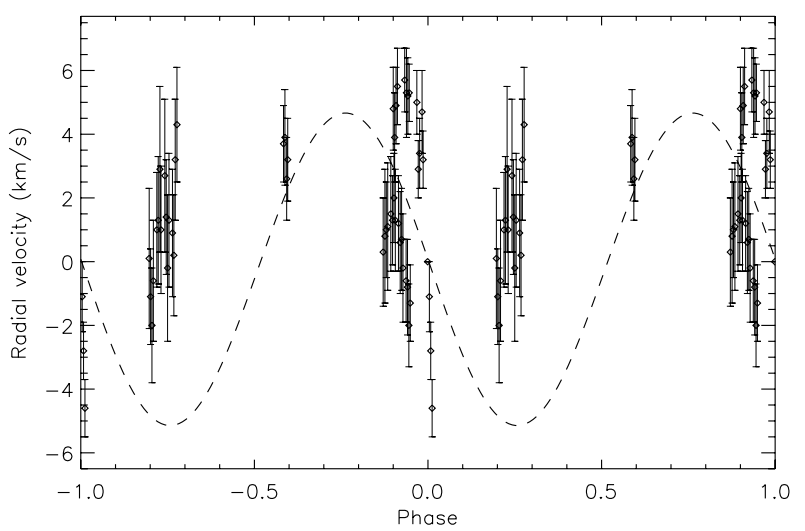

Fig. 10. Best primary RV curve fit for PHOEBE model 2 (parameters as for Fig. 9).

J2344's spectrum, the strongest absorption lines (H $\alpha$ and $\mathrm{Na}$ I D) were consistently visible, but showed no signs of shifting or splitting. Also, the SuperWASP archive contains a light curve for object b (1SWASP J234403.11-212205.8) which we obtained and analysed; in the 3.5 pixel aperture it showed similar variability to $\mathrm{J} 2344$, but in the smaller 2.5 pixel aperture, which should have excluded most of its neighbour's flux, its variability was less periodic, while J2344's light curve exhibited the same periodic behaviour even in the small aperture. Finally, D. Boyd confirmed from his 18 December 2012 observations of the field of view that object b had an (unfiltered) magnitude of $15.46 \pm 0.21$, corresponding to a SuperWASP flux variation of only about \pm 0.3 units: far smaller than J2344's amplitude of \pm 1 unit. He also noted that object $\mathrm{c}$ did not surpass his sky background level of 16.5-17.0 mag; it therefore would have been too faint to be detectable by SuperWASP, with its range of $\sim 8-15 \mathrm{~V}$ mag. We must conclude, then, that $\mathrm{J} 2344$ really is the source of the periodic photometric variation observed.

What, then, might explain an object with the light curve of a short-period binary but the spectrum of a single star? Moreover, what is the source of its dramatic period changes, with their 4.19 y meta-cycle? We have explored three physical models which provide potential explanations for these observations.

\subsection{One-star model}

Our first model regards J2344 as what its spectrum indicates: a single mid-K dwarf, rotating with a period of $18461.6 \mathrm{~s}$. The
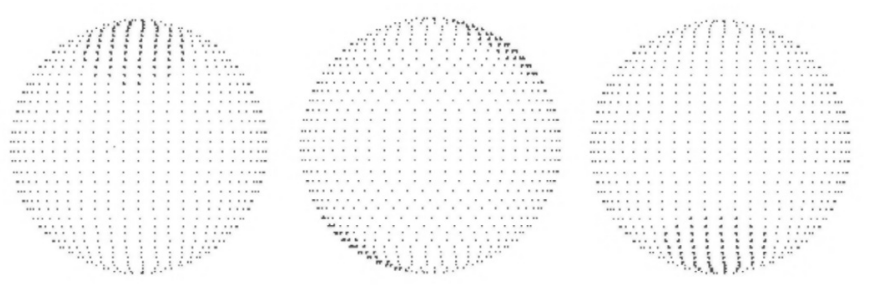

Fig. 11. PHOEBE images of spotted star model at phases $0.0,0.25$ and 0.5 , from left to right.

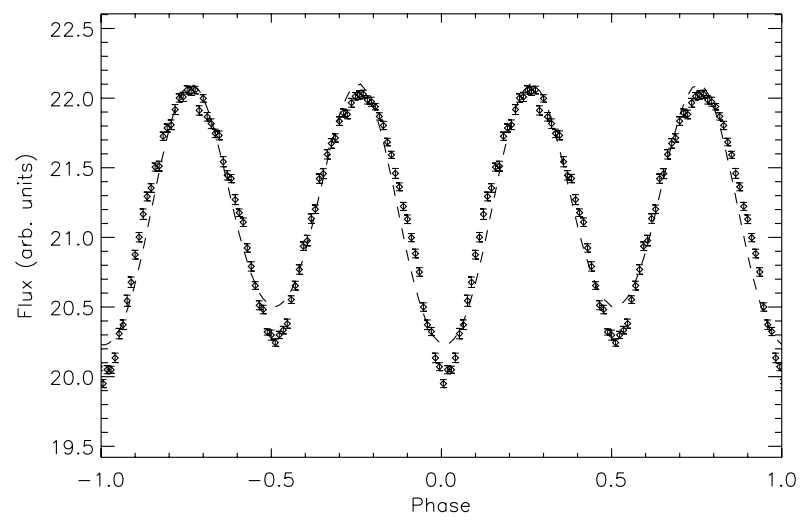

Fig. 12. Best light curve fit for PHOEBE spotted star model.

low amplitude of light curve variability would be consistent with rotational variation caused by cool surface spots. However, the alternating deeper and shallower minima at phases 0.0 and 0.5 (Fig. 2), observed over many years of ASAS and SuperWASP data, would require two large stable spots of different areas and/or temperatures, located on diametrically-opposite sides of the star (Fig. 11). This could be achieved if the spots were somehow pinned to the star's magnetic poles (Harrison et al. (2012) claimed similar cool stable polar spots on many K-class rotational variables observed with Kepler), and if the star were an oblique rotator (Stibbs 1950), having its magnetic axis at an angle to its axis of rotation. The small RV excursions from zero would then be caused by a form of the Rossiter-McLaughlin effect (Rossiter 1924; McLaughlin 1924) associated with the spots, as observed by Huber et al. (2009). Precession due to the different alignments of magnetic and rotational axes might explain the $4.19 \mathrm{y}$ meta-cycle of period changes (Monaghan 1968).

Using PHOEBE again to test this idea, we modelled a single rotational variable in similar fashion to Harrison et al., setting the input orbital period to the assumed rotational period and turning off the light from the detached companion. Since we were interested in reproducing the RVs as well as the light curve, we set the mass ratio as low as possible so that the modelled curves were both flat before the introduction of spots. The (primary) star was given a mass and effective temperature consistent with a $\mathrm{K} 5 \mathrm{~V}$ spectrum, and $i$ was set to $90^{\circ}$ for simplicity. Two spots were then added to the primary in accordance with the model, and adjusted manually until reasonable light and RV curve fits were obtained. The final spot location parameters were colatitudes $35^{\circ}$ and $145^{\circ}$ and longitudes $0^{\circ}$ and $180^{\circ}$ respectively, to simulate stable location on the poles of a magnetic axis at $35^{\circ}$ to the rotational axis. One was given a slightly larger radius $\left(26^{\circ}\right.$ vs. $\left.25^{\circ}\right)$ to reproduce the different depths of light curve minima, but both were set to the same temperature (20\% of average).

Figures 12 and 13 show the resulting fits. The light curve is fairly well reproduced, both in amplitude and different minima 


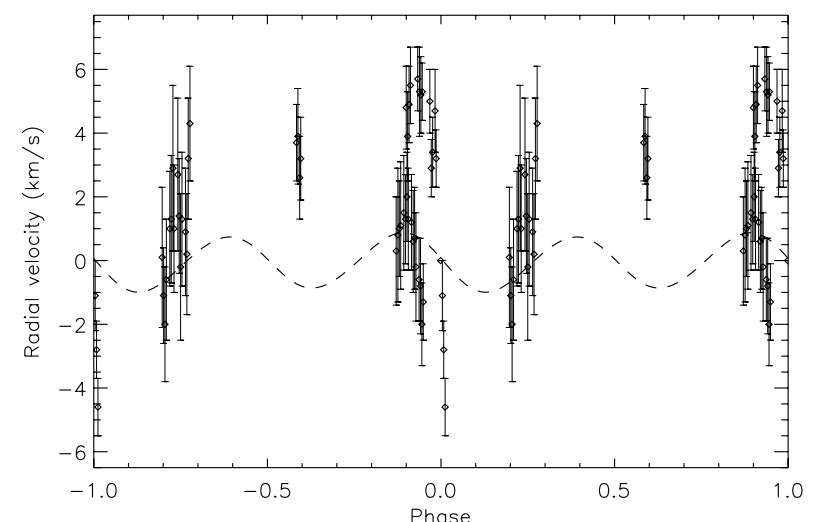

Fig. 13. Best RV curve fit for PHOEBE spotted star model.

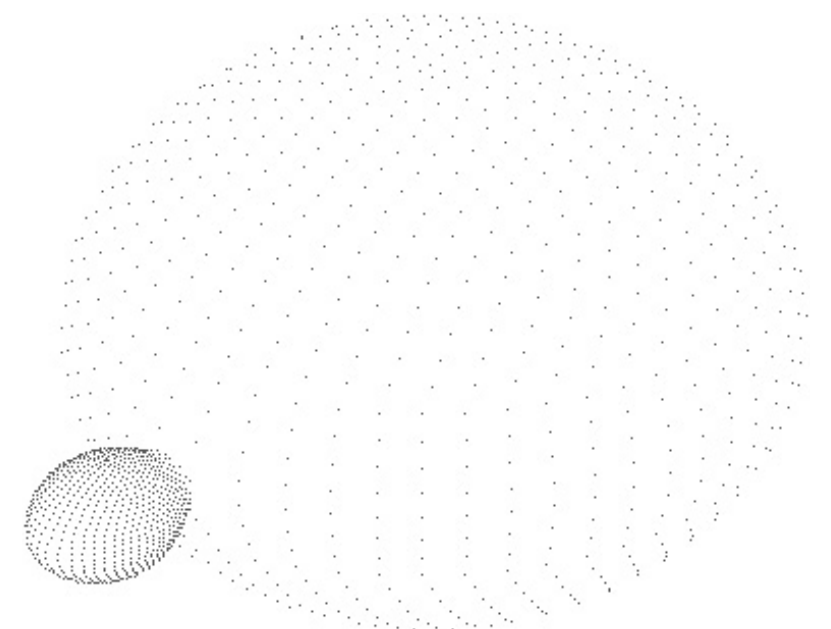

Fig. 14. PHOEBE image of $K$ dwarf+brown dwarf binary model at phase 0.9 .

depths. The fitted RV curve is somewhat smaller in amplitude than that observed, but does match some of the velocity trends in the observed curve, notably the maximum around phase 0.9 and the rising trend around phase 0.25 . Better fits might be obtained by modelling non-spherical spots, but these initial results may at least serve as proof of concept. A greater problem for the onestar model is the lack of evidence (to our knowledge) for other low-mass oblique rotators.

\subsection{Two-star model}

Our second model for J2344 takes the light curve at face value, seeing it as an eclipsing binary in contact configuration, with a mid-K dwarf as primary, and a secondary component making a very limited contribution to the spectrum. In order to reproduce the observed amplitude of RV variation (associated with the primary, in this model), the secondary's mass must be in the brown dwarf range (Fig. 14). The observed shape of the RV curve would then be due to the Rossiter-McLaughlin effect as the secondary obscures each side of the primary in turn, and the $4.19 \mathrm{y}$ meta-cycle of period changes could be explained by the Applegate mechanism (Applegate 1992).

A PHOEBE model with $i=59^{\circ}, q=0.025, M_{1}=0.79 M_{\odot}$ and $M_{2}=0.02 M_{\odot}$ produced an excellent fit to the observed light curve amplitude and shape, though the different depths

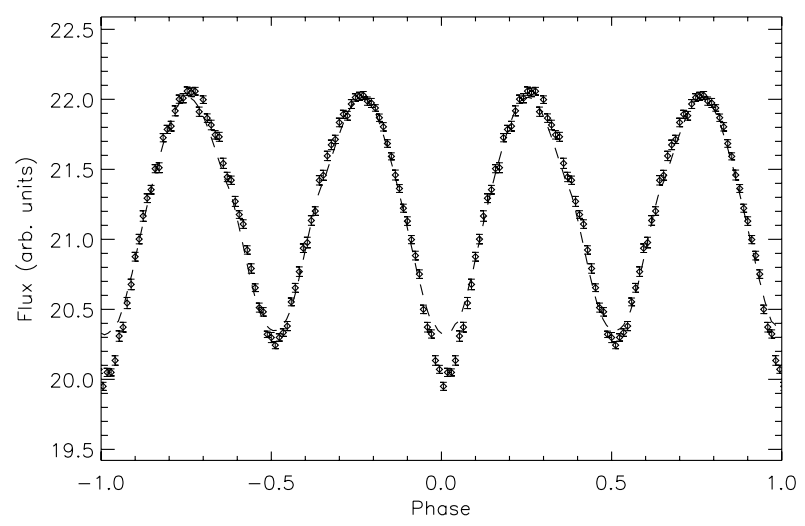

Fig. 15. Best light curve fit for PHOEBE K dwarf+brown dwarf binary model.

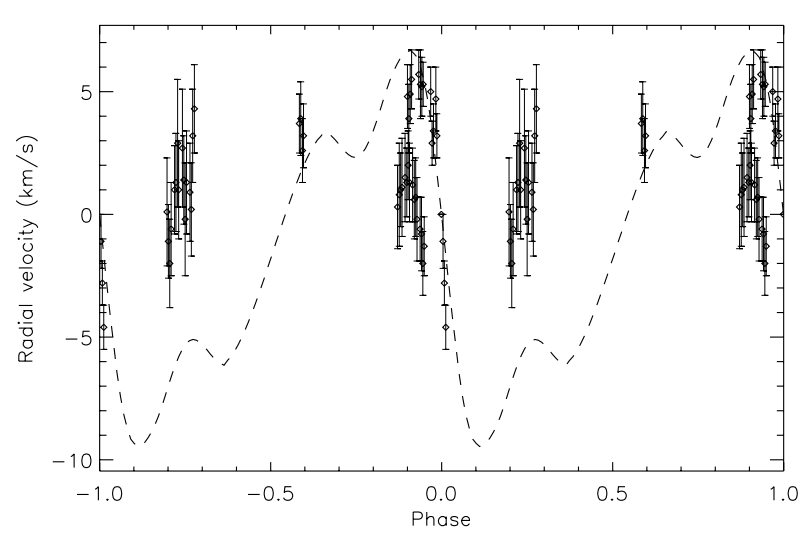

Fig. 16. Best primary RV curve fit for PHOEBE K dwarf+brown dwarf binary model.

of minima could not be easily reproduced (Fig. 15). The RV fit (Fig. 16) was of slightly greater amplitude than the observed curve, but its Rossiter-McLaughlin effect-induced variations matched the velocity trends reasonably well, as with the spotted star model. We note, however, that PHOEBE is not intended to model planetary-mass companions, and may not model well objects in the brown dwarf range either, so these model outputs should be regarded with caution.

\subsection{Three-star model}

Our final model for J2344 is of a triple system, consisting of a very low-mass contact eclipsing binary orbiting a more massive mid-K star which dominates the spectrum and obscures the contribution from the binary. The light curve is then the sum of a constant flux contribution from the K star (providing up to 9/11 of the maximum system flux), and a periodically-variable contribution from the binary. The RV curve is almost constant, since it largely represents the unvarying (on this timescale) position of the K dwarf. The 4.19 y cycle of period variation would be a light-time effect (LITE) resulting from the orbit of the contact binary around the most massive component of the triple.

A third light can be readily included in PHOEBE's models; Figs. 17 to 19 show the results of modelling the eclipsing binary in such a triple system, using parameters $i=77^{\circ}, q=0.5$, $M_{1}=0.34 M_{\odot}$ and $M_{2}=0.17 M_{\odot}$ (i.e. $M$ dwarfs), and a maximal third light of 18.0 SuperWASP flux units (11.86 V mag). 


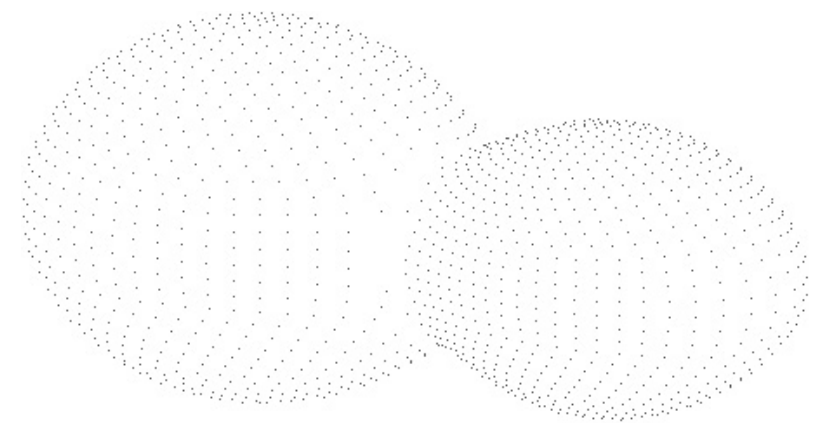

Fig. 17. PHOEBE image of $\mathrm{M}+\mathrm{M}$ dwarf binary model at phase 0.15 .

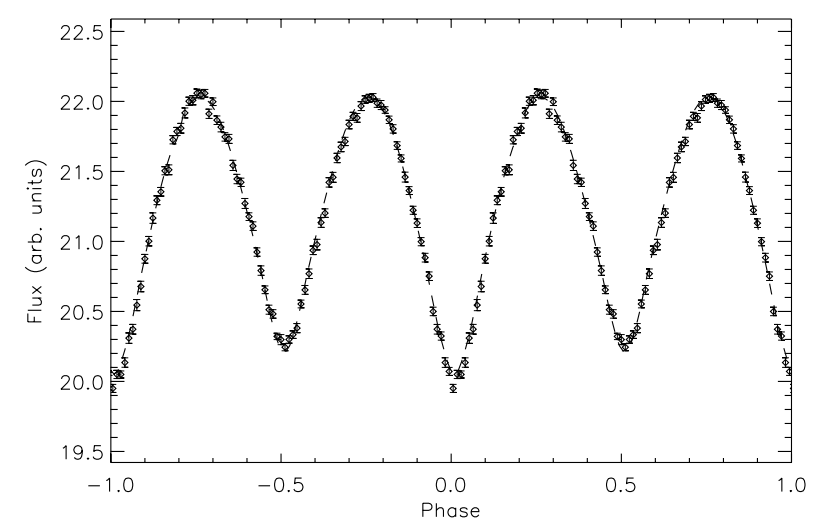

Fig. 18. Best light curve fit for PHOEBE $\mathrm{M}+\mathrm{M}$ dwarf binary model.

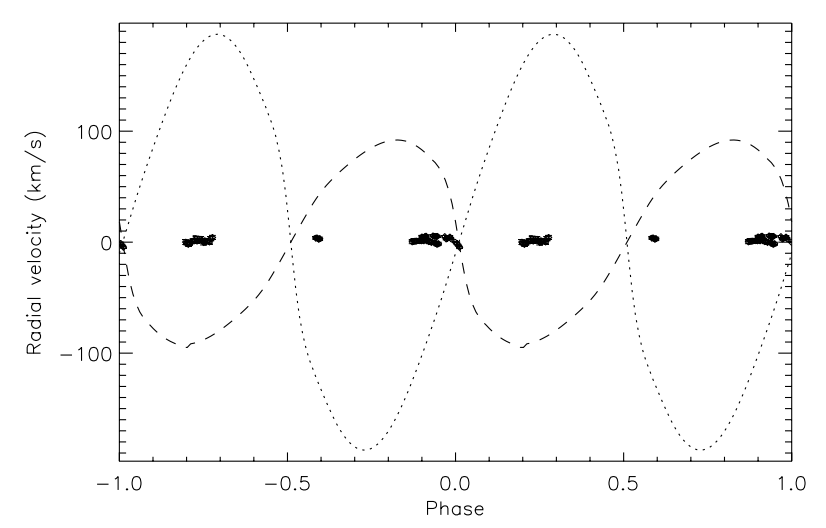

Fig. 19. RV curves corresponding to best light curve fit for PHOEBE $\mathrm{M}+\mathrm{M}$ dwarf binary model (primary curve shown with dashed line; secondary with dotted line.)

Figure 17 indicates the very deep contact required; assuming this, however, an excellent fit to the light curve is achieved, without even needing spots to be included for fine-tuning (Fig. 18). Figure 19 shows the primary and secondary RV curves implied for such an eclipsing binary, for reference only, since our observed SALT velocities are expected to be dominated by the constant $\mathrm{K}$ star, which is not included in the PHOEBE model as a mass, only as a light source.

Using these masses, totalling $\sim 0.5 M_{\odot}$, for the binary, and a plausible $0.65 M_{\odot}$ for the K5 system primary, a binary-toprimary flux ratio of about 1:5 is implied, consistent with the modelled ratio of around 1:6 given by the SuperWASP light curve with maximum third light. We can also insert these values

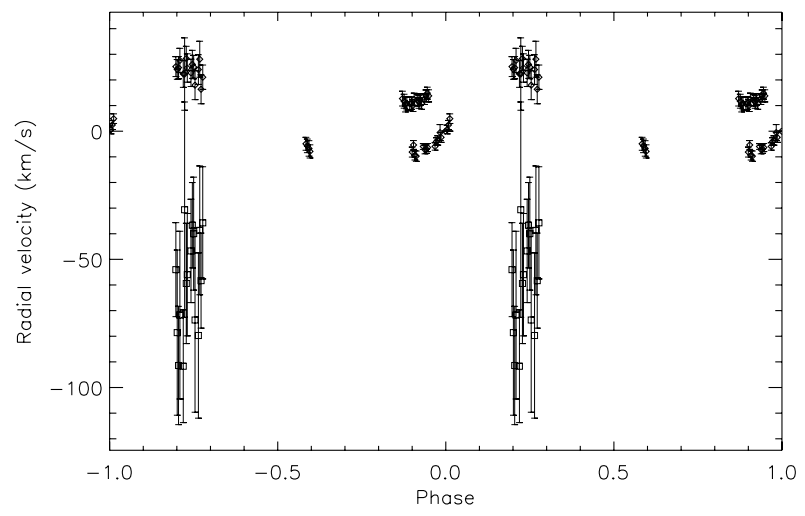

Fig. 20. Residual RV curves for J2344, following subtraction of scaled $\mathrm{K} 5 \mathrm{~V}$ template spectrum, and cross-correlation with phase 0 residual spectrum. The stronger cross-correlation peak velocities are shown with diamonds; the fainter cross-correlation peak visible near quadrature is plotted with squares.

into the approximate formula for expected LITE changes for a binary in an edge-on circular orbit with a third body, given by Pribulla et al. (2012) in their Eq. (5):

$\Delta T \approx \frac{2 M_{3} G^{1 / 3}}{c}\left[\frac{P_{3}}{2 \pi\left(M_{1}+M_{2}\right)}\right]^{2 / 3}$,

where $M_{1,2}$ are the masses of the binary components, $M_{3}$ is the mass of the $\mathrm{K} 5$ system primary, $P_{3}$ is the 4.19 y period of $\mathrm{O}-\mathrm{C}$ oscillations, and $\Delta T$ is the peak-to-peak amplitude of the $\mathrm{O}-\mathrm{C}$ variations. The result is $2640 \mathrm{~s}$, about twice the observed amplitude (Fig. 3), which is entirely plausible if we do not expect the angle of inclination to be $90^{\circ}$. (Indeed, using Pribulla et al.'s Eq. (10) with these masses and our observed semi-amplitude of LITE of $631 \mathrm{~s}, i=56^{\circ}$ is suggested.)

On the assumption that this model was approximately correct, a suitably-scaled constant K5 template spectrum was subtracted from each of our spectra to see whether some trace of an $\mathrm{M}+\mathrm{M}$ eclipsing binary spectrum might be detectable in the residuals. Figure 20 shows the resulting RV curves, after crosscorrelation with a phase 0 residual spectrum. A second crosscorrelation peak was now marginally detectable in the spectra near quadrature, yielding RVs in a similar range, and following a similar upward trend, to those predicted for the primary curve in Fig. 19 near phase 0.25. The other RVs reached greater amplitudes than before (Fig. 6) and might conceivably represent a blending of lines from multiple stellar components. Given that our best model for the low-mass eclipsing binary in this putative triple system involves very deep contact, it is likely that their lines would be significantly broadened and blended even if no third spectrum were present to complicate the picture, making extraction of RVs challenging in any case.

2MASS and WISE colours for the source were also checked for evidence of an infrared excess which might support the presence of M dwarfs in the system. The results were inconclusive, however: 2MASS $J-H$ and $H-K$ colours were in ranges expected for a K5 star, while the WISE colours were inconsistent, possibly being contaminated by nearby sources.

Although we lack conclusive evidence for it, a triple system seems the most likely of our three models for J2344. We have not assessed the dynamical stability of such a triple, but note the recent detection of a young hierarchical triple composed of a late-K primary and a pair of mid-M dwarfs in wide orbits 
(Deacon et al. 2013) which shows some similarity to the system posited here. Using the observed and theoretical absolute magnitudes for the stars in this model, a distance of 80-90 pc is indicated. The calculated separation between system primary and contact binary is $2.7 \mathrm{AU}$, which would then correspond to an angular separation of $\sim 0$ '.03, making the components resolvable in principle. The expected RV amplitude for the system primary, over a 4.19 y orbital period with the binary, would be around $8 \mathrm{~km} \mathrm{~s}^{-1}$ (assuming a circular orbit and the same angle of inclination as for the contact binary), which might also be detectable in the long term.

\section{Conclusions}

Object J2344, which we originally thought might be an eclipsing binary close to stellar merger, has proved to be unusual and intriguing in a different way. Of the three models investigated here, a triple system containing a low-mass eclipsing binary appears the most plausible explanation for the apparently conflicting photometric and spectroscopic data, and provides an appealing reason for the dramatic cyclic variations in period length observed as well. If confirmed, it should provide a useful contribution to studies of multiple systems, alongside the quadruple doubly-eclipsing system already detected in our collection of short-period SuperWASP eclipsing binary candidates (Lohr et al. 2013a).

Alternatively, if one of our other explanations proves more likely, J2344 could add to our knowledge of brown dwarfs, or constitute a rare type of rotational variable. Still other explanations are no doubt conceivable e.g. involving higher multiplicity of the system; in any case, this appears to be an interesting object worthy of further observation. We would hope in the future to obtain multi-colour photometry and near-infrared spectroscopy of J2344, with improved phase coverage, in the expectation that the greater contrast available at longer wavelengths would increase the opportunity of detecting cool, low-mass objects within the system.
Acknowledgements. The WASP project is funded and operated by Queen's University Belfast, the Universities of Keele, St. Andrews and Leicester, the Open University, the Isaac Newton Group, the Instituto de Astrofisica de Canarias, the South African Astronomical Observatory and by STFC. Some of the observations reported in this paper were obtained with the Southern African Large Telescope (SALT) under program 2012-1-UKSC-007 (PI: Andrew Norton). This work was supported by the Science and Technology Funding Council and the Open University.

\section{References}

Applegate, J. H. 1992, ApJ, 385, 621

Buckley, D. A. H., Swart, G. P., \& Meiring, J. G. 2006, in Ground-based and Airborne Telescopes, ed. L. M. Stepp, Proc. SPIE, 6267, 32

Burgh, E. B., Nordsieck, K. H., Kobulnicky, H. A., et al. 2003, in Instrument Design and Performance for Optical/Infrared Ground-based Telescopes, eds. M. Iye, \& A. Moorwood, Proc. SPIE, 4841, 1463

Crawford, S. M., Still, M., Schellart, P., et al. 2010, in Observatory Operations: Strategies, Processes, and Systems III, eds. D. R. Silva, A. B. Peck, \& B. T. Soifer, Proc. SPIE, 7737, 54

Deacon, N. R., Schlieder, J. E., Olofsson, J., Johnston, K. G., \& Henning, T. 2013, MNRAS, 434, 1117

Harrison, T. E., Coughlin, J. L., Ule, N. M., \& López-Morales, M. 2012, ApJ, 143,4

Huber, K. F., Wolter, U., Czesla, S., et al. 2009, A\&A, 501, 715

Lohr, M. E., Norton, A. J., Kolb, U. C., et al. 2012, A\&A, 542, A124

Lohr, M. E., Norton, A. J., Kolb, U. C., et al. 2013a, A\&A, 549, A86

Lohr, M. E., Hodgkin, S. T., Norton, A. J., \& Kolb, U. C. 2013b, A\&A, submitted

Mazeh, T., Tamuz, O., Zucker, S., et al. 2006, in Tenth Anniversary of 51 Pegb: Status of and prospects for hot Jupiter studies. Colloquium held at Observatoire de Haute Provence, France, August 22-25, 2005, eds. L. Arnold, F. Bouchy, \& C. Moutou (Paris: Frontier Group), 165

McLaughlin, D. B. 1924, ApJ, 60, 22

Monaghan, J. J. 1968, Z. Astrophys., 69, 154

Norton, A. J., Payne, S. G., Evans, T., et al. 2011, A\&A, 528, A90

Pollacco, D. L., Skillen, I., Cameron, A. C., et al. 2006, PASP, 118, 1407

Pribulla, T., Vaňko, M., von Eiff, M. A., et al. 2012, Astron. Nachr., 333, 754

Prša, A., \& Zwitter, T. 2005, ApJ, 628, 426

Rossiter, R. A. 1924, ApJ, 60, 15

Rucinski, S. M. 1992, AJ, 103, 960

Stibbs, D. W. N. 1950, MNRAS, 110, 395

Tamuz, O., Mazeh, T., \& Zucker, S. 2005, MNRAS, 356, 1466

Valdes, F., Gupta, R., Rose, J. A., Singh, H. P., \& Bell, D. J. 2004, ApJS, 152, 251

Wilson, R. E., \& Devinney, E. J. 1971, ApJ, 166, 605 